\title{
The assessment of the effectiveness of extracorporeal shock wave therapy (ESWT) for soft tissue injuries (ASSERT): the future
}

Nicola Maffulli $\mathbf{N}^{1,2,3}$

Gayle Maffulli ${ }^{4}$

1 Department of Musculoskeletal Disorders, Faculty of Medicine and Surgery, University of Salerno,

Salerno, Italy

2 Institute of Science and Technology in Medicine, Keele University School of Medicine, Stoke on Trent, UK

3 Centre for Sports and Exercise Medicine, Queen Mary University of London, Barts and the London School of Medicine and Dentistry, Mile End Hospital, London, UK

4 Wholelife Clinics, London, UK

Corresponding author:

Nicola Maffulli

Department of Musculoskeletal Disorders

School of Medicine and Surgery

University of Salerno,

Mary University of London

Barts and the London School of Medicine and Dentistry

Centre for Sports and Exercise Medicine

Mile End Hospital 275 Bancroft Road

E1 4DG, London, England, UK

Tel.: +447989358279

E-mail:n.maffulli@qmul.ac.uk

\section{Summary}

Introduction: The online database for the Assessment of Effectiveness of Extracorporeal Shock Wave Therapy for Soft Tissue Injuries (ASSERT) successfully collected prospective information on the effectiveness of ESWT across the UK in a standardised fashion. ASSERT has produced remarkable results, applicable to the population at large, not just to the selected few patients who are carefully entered in randomised controlled trial. During the collection, follow-up and analysis, it become evident how important it was to continue to work within the framework of ASSERT to collect unique data. By the same token, we realised that not everything worked in an optimal fashion. ASSERT 2 was planned, and it will involve more conditions and a more streamlined approach to data collection and analysis.
KEY WORDS: chronic soft tissue injuries, extracorporeal shock wave therapy, effectiveness, short and long-term effects.

\section{Introduction}

Extracorporeal shock wave therapy (ESWT) is safe and effective in the non-operative management of soft tissue musculoskeletal ailments.

The clinical effectiveness of ESWT was considered controversial ${ }^{1}$, and the National Institute for Health and Clinical Excellence (NICE) gave guidance on the use of ESWT ${ }^{2-6}$. NICE recommended that the results of ESWT are monitored, and clinicians undertaking the procedure make special arrangements for audit.

The Assessment of Effectiveness of Extracorporeal Shock Wave Therapy (ESWT) for Soft Tissue Injuries (ASSERT) database started to collect data in 2011, and the last patient was recruited in 2014. We needed to collect two-year outcome data on these patients, suffering from refractory plantar fasciopathy, tennis elbow, Achilles tendinopathy, greater trochanter pain syndrome, patellar tendinopathy, and it took until 2016 before all the relevant outcome data were collated.

ASSERT collected information on the effectiveness of ESWT across the UK using standard ESWT equipment and a standardised treatment protocol, together with standardised baseline measurements and outcome measures and time points in centres across the UK. There is no doubt that ASSERT has allowed a unique picture of the use and outcome of ESWT in soft tissue musculoskeletal disorders in a real life situation. Nevertheless, by the end of 2014, we did realise that, no matter how perfect we thought ASSERT was when we planned it, patient recruitment and data collection could have been performed in a more rational and streamlined fashion, using more modern systems.

In the spirit of the original ASSERT, and following the same principles, in 2014 we started ASSERT 2.

Hopefully, we will be able to analyse and write up the relevant data soon.

We hope that the results of ASSERT have been compelling, and they helped to advance the science and practice of shock wave therapy in musculoskeletal disorders.

Watch this space for ASSERT 2! 


\section{Compliance with ethical standards}

\section{Conflict of interest}

All Authors declare no conflict of interest.

\section{Funding}

The ASSERT database has been developed and established through funds provided by Industry (Spectrum Technology UK) and ESPRC grants.

\section{Acknowledgements}

We thank Mr Jim Westwood and Mr Chris Schiel from Spectrum Technology for their support. Mr Nathan Bentley of twotwentyseven London Ltd - a creative digital agency developed the ASSERT platform following the direction of Professor Nicola Maffulli and Mrs Gayle Maffulli.

We thank all the clinicians recruiting participants onto the ASSERT database and the participants of ASSERT. Professor Nicola Maffulli developed the concept of ASSERT.

\section{Ethical approval}

All procedures performed in this study involving human participants were in accordance with the ethical standards of the institutional and/or national research committee and with the 1964 Helsinki Declaration and its later amendments or comparable ethical standards.

\section{Informed consent}

Informed consent was obtained from all individual participants included in the study.

\section{Ethics}

The Authors declare that this research was conducted following basic ethical aspects and international standards as required by the journal and recently update in?

\section{References}

1. Rompe JD, Maffulli N. Repetitive shock wave therapy for lateral elbow tendinopathy (tennis elbow): a systematic and qualitative analysis. B Med Bull. 2007:1-24.

2. National Institute for Health and Clinical Excellence (NICE). Extracorporeal shock wave therapy for calcific tendonitis of the shoulder. 2003, N0354. www.nice.org.uk

3. National Institute for Health and Clinical Excellence (NICE). Extracorporeal shock wave therapy for refractory plantar faciitis. 2009, N1972. www.nice.org.uk

4. National Institute for Health and Clinical Excellence (NICE). Extracorporeal shock wave therapy for refractory tennis elbow. 2009, N1976. www.nice.org.uk

5. National Institute for Health and Clinical Excellence (NICE). Extracorporeal shock wave therapy for refractory Achilles tendinopathy. 2009, N1973. www.nice.org.uk

6. National Institute for Health and Clinical Excellence (NICE). Extracorporeal shock wave therapy for refractory greater trochanter pain syndrome. 2011, N2143. www.nice.org.uk

7. Padulo J, Oliva F, Frizziero A, Maffulli N. Muscles, Ligaments and Tendons Journal - Basic principles and recommendations in clinical and field science research: 2016 update. MLTJ. 2016;6(1):1-5. 